\title{
APPENDIX III. COMPOSITION AND SOURCE OF DETRITAL SAND LAYERS FROM THE GUAYMAS BASIN ${ }^{1}$
}

J. Eduardo Aguayo, Instituto Mexicano del Petróleo, México 14, D.F., Mexico

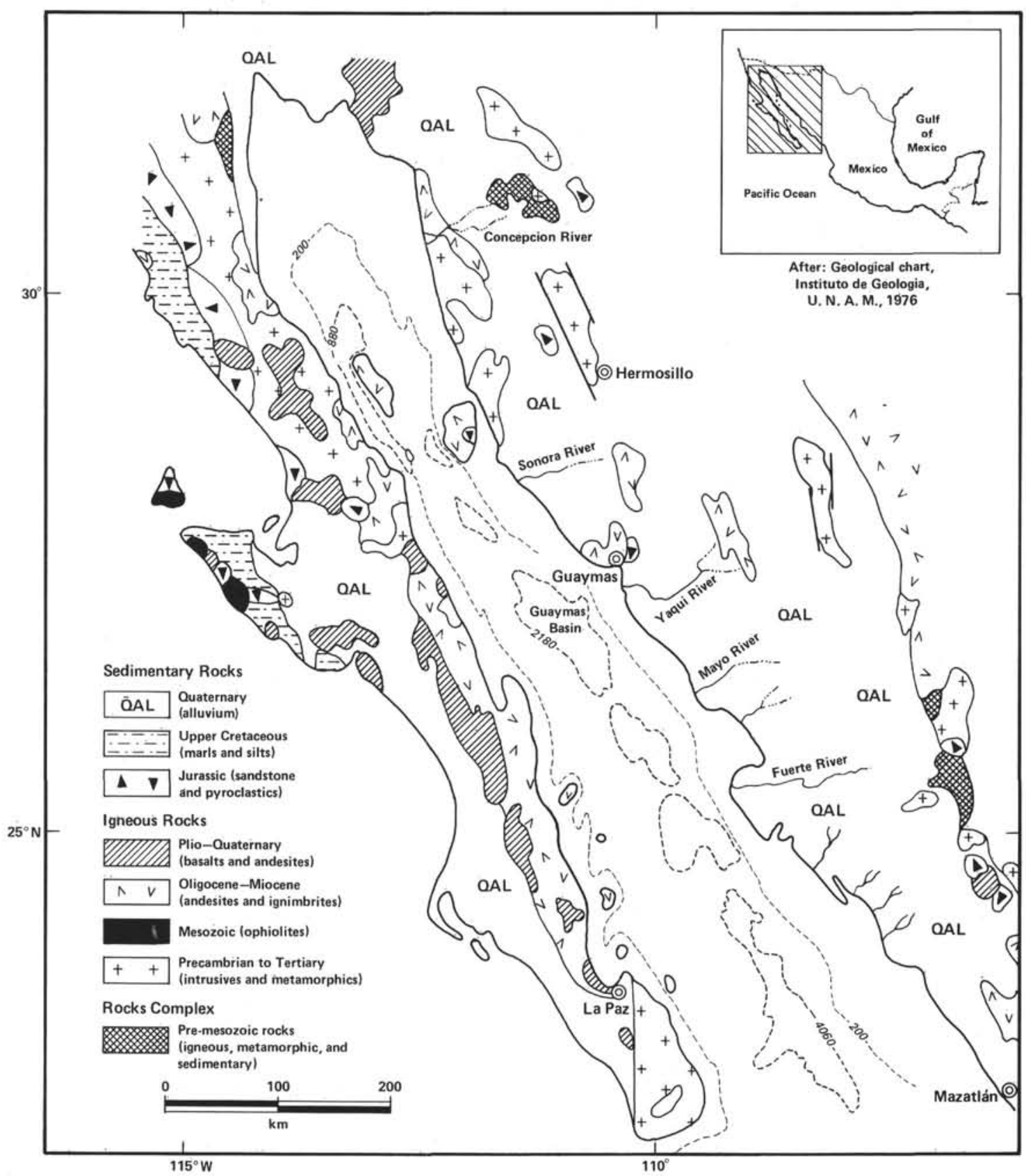

Figure 1. Generalized distribution of lithological facies in northwest Mexico.

${ }^{1}$ Curray, J. R., Moore, D. G., et al., Init. Repts. DSDP, 64: Washington (U.S. Govt. Printing Office). 

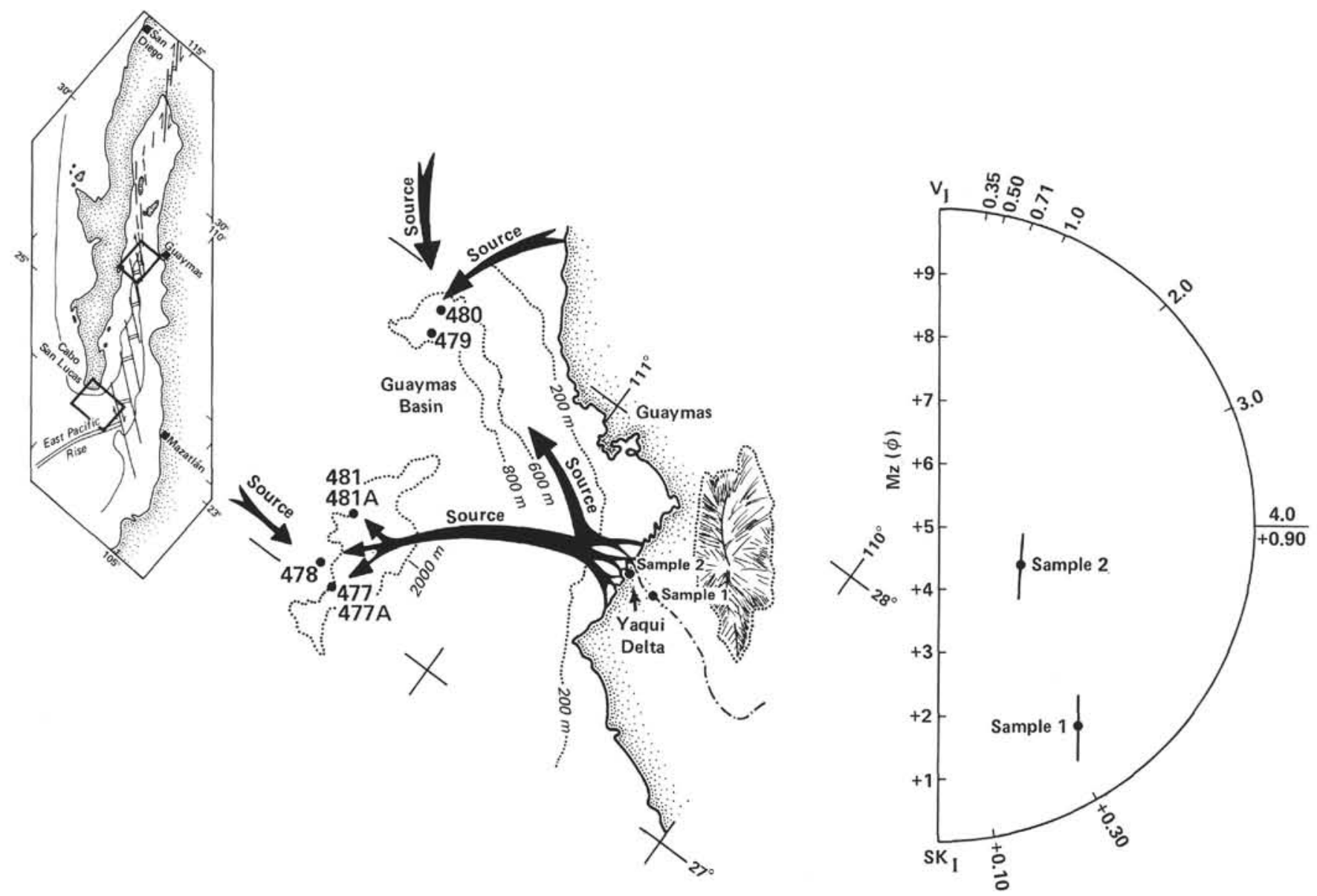

\begin{tabular}{|c|c|c|c|c|c|c|c|c|c|c|c|c|c|c|c|c|c|}
\hline \multirow[b]{2}{*}{ Sample } & \multirow[b]{2}{*}{ Description } & \multicolumn{16}{|c|}{ Composition (\%) } \\
\hline & & $\mathrm{Mz}(\phi)$ & $\mathrm{V}_{\mathrm{I}}$ & $\mathrm{SK}_{\mathrm{I}}$ & $\mathrm{Qz}$ & IRF & Fs & Aug & Hor & Hyp & $\mathrm{Mi}$ & Oliv & Apat & Epid & Mag & Tit & $\mathrm{IIm}$ \\
\hline 1 & $\begin{array}{l}\text { Dark greenish gray } \\
\text { (5G } 4 / 1) \text { medium sand, } \\
\text { poorly sorted, fine. } \\
\text { skewed. }\end{array}$ & 1.75 & 1.25 & +0.28 & 25 & 20 & 15 & 5 & 5 & 3 & 10 & 3 & 3 & 5 & 3 & 3 & \\
\hline 2 & $\begin{array}{l}\text { Medium gray (N5) coarse } \\
\text { sandy silt, moderately } \\
\text { sorted, fine-skewed. }\end{array}$ & 4.30 & 0.81 & +0.12 & 45 & & 30 & & 2 & & 10 & & 5 & 3 & 3 & & 2 \\
\hline
\end{tabular}

Note: $M z(\phi)=$ mean size; $V_{1}=$ sorting; $S K_{I}=$ skewness; Apat = apatite; $A u g=$ augite; Dol = dolomite; Epid = epidote; Fs = feldspar; Hem = hematite; Hor = hornblende; $\mathrm{Hyp}=$ hypersthene; IIm = ilmenite; IRF = igneous rock fragments; Mag = magnetite; Mi = mica; Oliv = olivine; $\mathrm{Py}=$ pyrite; $\mathrm{Qz}=$ quartz; VG = volcanic glass; Zeol = zeolite; Diat = diatoms; Rads = radiolarians; Fors = foraminifers; Sp Spic = sponge spicules.

Figure 2. Source areas of sediments deposited in the Guaymas Basin and textural attributes of Yaqui River sediments. 

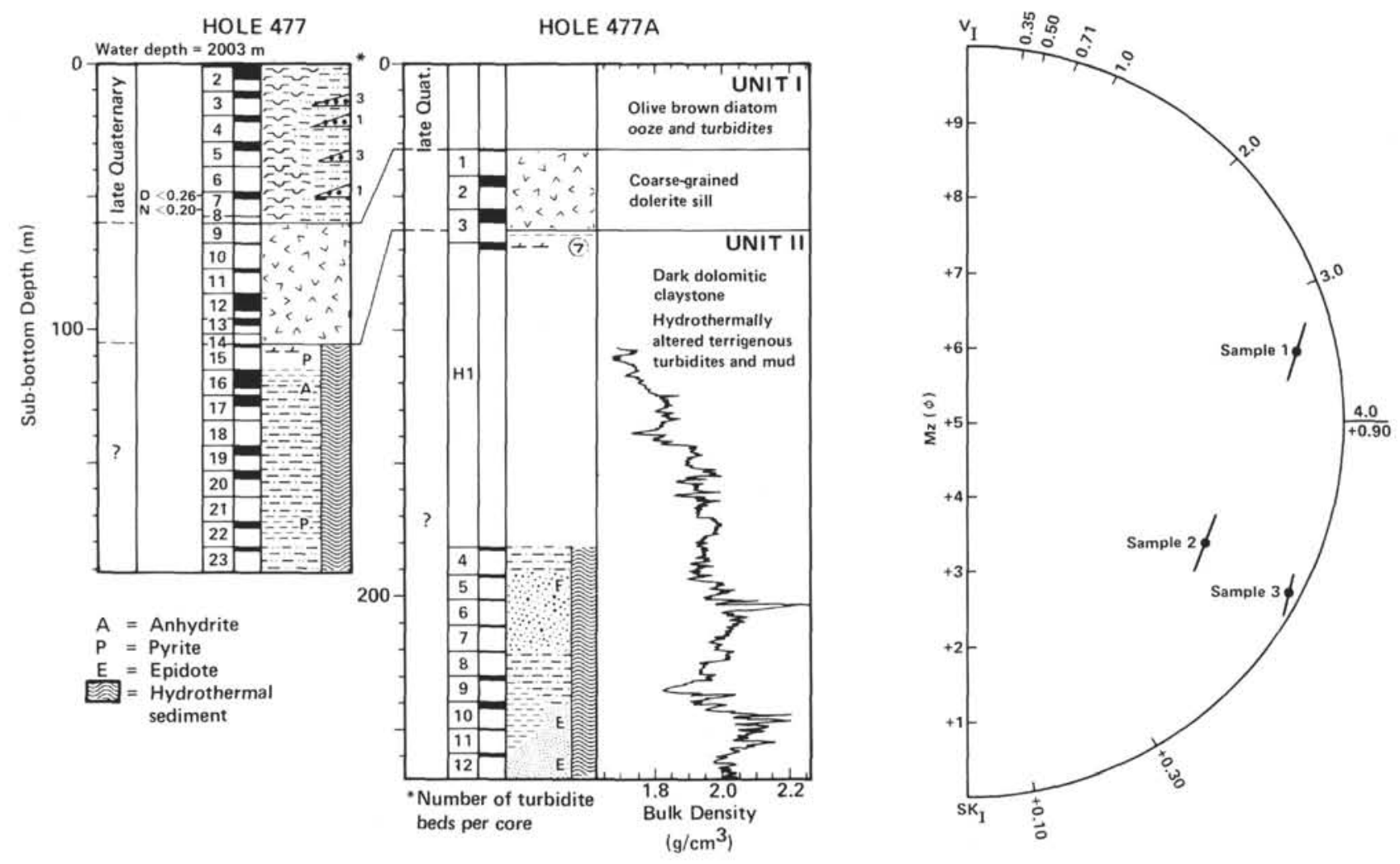

\begin{tabular}{|c|c|c|c|c|c|c|c|c|c|c|c|c|c|c|c|c|c|c|c|}
\hline \multirow[b]{2}{*}{ Sample } & \multirow{2}{*}{$\begin{array}{l}\text { Core/Section } \\
\text { (interval in cm) }\end{array}$} & \multirow[b]{2}{*}{ Description } & \multicolumn{17}{|c|}{ Composition (\%) } \\
\hline & & & $\operatorname{Mz}(\phi)$ & $v_{1}$ & $\mathrm{SK}_{\mathrm{I}}$ & $\mathrm{Oz}$ & Fs & $\mathrm{Mi}$ & Aug & Py & Epid & VG & Horn & Zeol & $\mathrm{Hem}$ & Diat & Rads & Fors & Sp Spic \\
\hline 1 & $\begin{array}{l}477 \\
4-1,100-102\end{array}$ & $\begin{array}{l}\text { Grayish olive (10Y } 4 / 2) \\
\text { sandy diatomaceous } \\
\text { silt, very poorly sorted, } \\
\text { strongly fine-skewed. }\end{array}$ & 6.0 & 3.10 & +0.32 & 5 & 2 & 2 & 1 & 5 & & & & & & 45 & 15 & 10 & 15 \\
\hline 2 & $\begin{array}{l}477 A \\
5-4,106-108\end{array}$ & $\begin{array}{l}\text { Medium light gray } \\
\text { (N6-5) very fine silty } \\
\text { sand, poorly sorted, } \\
\text { fine-skewed. }\end{array}$ & 3.43 & 2.41 & +0.23 & 30 & 35 & & & 2 & 15 & 10 & & & & & & 8 & \\
\hline 3 & $9-1,53-55$ & $\begin{array}{l}\text { Medium gray (N5) fine } \\
\text { silty sand, very poorly } \\
\text { sorted, strongly fine- } \\
\text { skewed. }\end{array}$ & 2.70 & 3.55 & +0.57 & 40 & 20 & & & 2 & 10 & & 10 & 15 & 3 & & & & \\
\hline
\end{tabular}

Figure 3. Mineralogical and textural attributes of terrigenous sediments from Holes 477 and 477A. Locations given on stratigraphic column. Note Samples 2 and 3 are from hydrothermally altered sands. (See Fig. 2 for explanation of symbols and abbreviations.) 

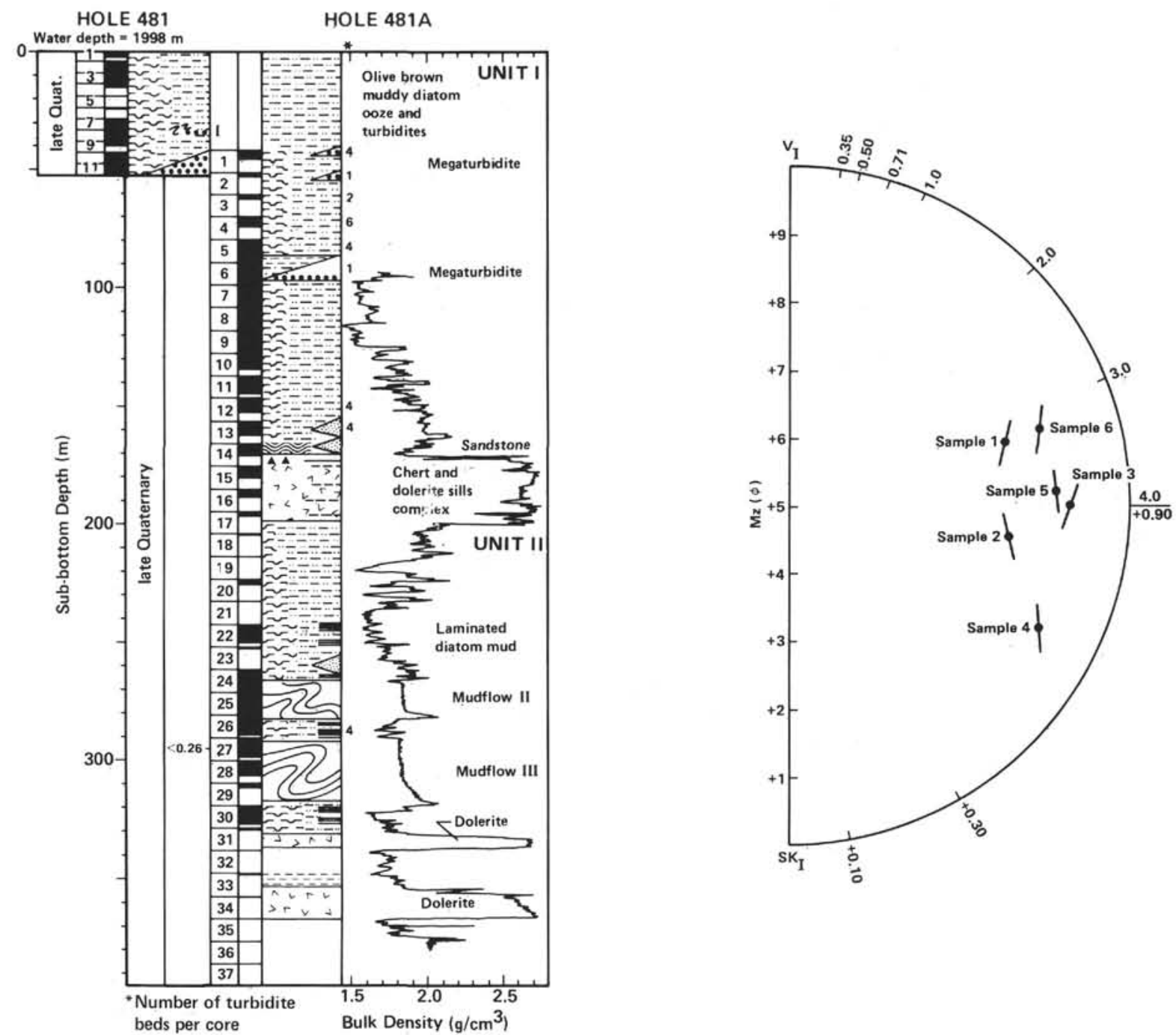

\begin{tabular}{|c|c|c|c|c|c|c|c|c|c|c|c|c|c|c|c|c|c|c|c|}
\hline \multirow[b]{2}{*}{ Sample } & \multirow{2}{*}{$\begin{array}{c}\text { Core/Section } \\
\text { (interval in cm) }\end{array}$} & \multirow[b]{2}{*}{ Description } & \multicolumn{17}{|c|}{ Composition (\%) } \\
\hline & & & $M z(\phi)$ & $v_{1}$ & $\mathrm{SK}_{\mathrm{I}}$ & $\mathrm{Oz}$ & Fs & $P y$ & Hor & Aug & Clay & IRF & Mi & VG & Others & Diat & Rads & Fors & Sp Spic \\
\hline 1 & $1-1,60-62$ & $\begin{array}{l}\text { Grayish olive (10Y } 4 / 1) \\
\text { medium diatomaceous } \\
\text { clayey silt, very poorly } \\
\text { sorted, fine-skewed. }\end{array}$ & 6.0 & 2.10 & +0.25 & 3 & 5 & 2 & 1 & 1 & 40 & & & & & 30 & 10 & 3 & 5 \\
\hline 2 & $1-2,96-98$ & $\begin{array}{l}\text { Grayish olive (10Y } 4 / 2) \\
\text { coarse diatomaceous } \\
\text { clayey silt, strongly } \\
\text { fine-skewed. }\end{array}$ & 4.5 & 1.25 & +0.50 & 10 & 20 & 3 & & & 40 & 5 & & & 1 & 20 & & 1 & \\
\hline 3 & $2-1,113-115$ & $\begin{array}{l}\text { Grayish olive (10Y } 4 / 2 \text { ) } \\
\text { sandy diatomaceous } \\
\text { clayey silt, very } \\
\text { poorly sorted, fine- } \\
\text { skewed. }\end{array}$ & 5.0 & 3.2 & +0.30 & 10 & 15 & 2 & 1 & & 45 & 2 & 2 & & 3 & 15 & 1 & 1 & 3 \\
\hline 4 & $6-4,63-65$ & $\begin{array}{l}\text { Grayish olive (10Y } 4 / 2 \text { ) } \\
\text { very fine silty sand, } \\
\text { poorly sorted, } \\
\text { strongly fine-skewed. }\end{array}$ & 3.2 & 1.8 & +0.50 & 30 & 35 & 1 & & 2 & & 2 & 3 & & 5 & 15 & 2 & 5 & \\
\hline 5 & $6-5,78-80$ & $\begin{array}{l}\text { Grayish olive (10Y 4/2) } \\
\text { medium sandy silt, } \\
\text { very poorly sorted, } \\
\text { strongly fine-skewed. }\end{array}$ & 5.26 & 2.11 & +0.57 & 30 & 35 & 3 & 2 & & & 1 & 2 & & 5 & 20 & 1 & 1 & \\
\hline 6 & $12-3,19-21$ & $\begin{array}{l}\text { Brown gray (5YR } 3 / 1) \\
\text { medium sandy clayey } \\
\text { silt, very poorly } \\
\text { sorted, strongly } \\
\text { fine-skewed. }\end{array}$ & 6.2 & 2.30 & +0.40 & 20 & 25 & & 5 & 5 & 20 & 7 & 3 & 3 & 15 & 3 & 2 & 5 & \\
\hline
\end{tabular}

Figure 4. Mineralogical and textural attributes of terrigenous sediments from Hole 481A. Note uniform source and abundance of feldspar. (See Fig. 2 for explanation of symbols and abbreviations.) 

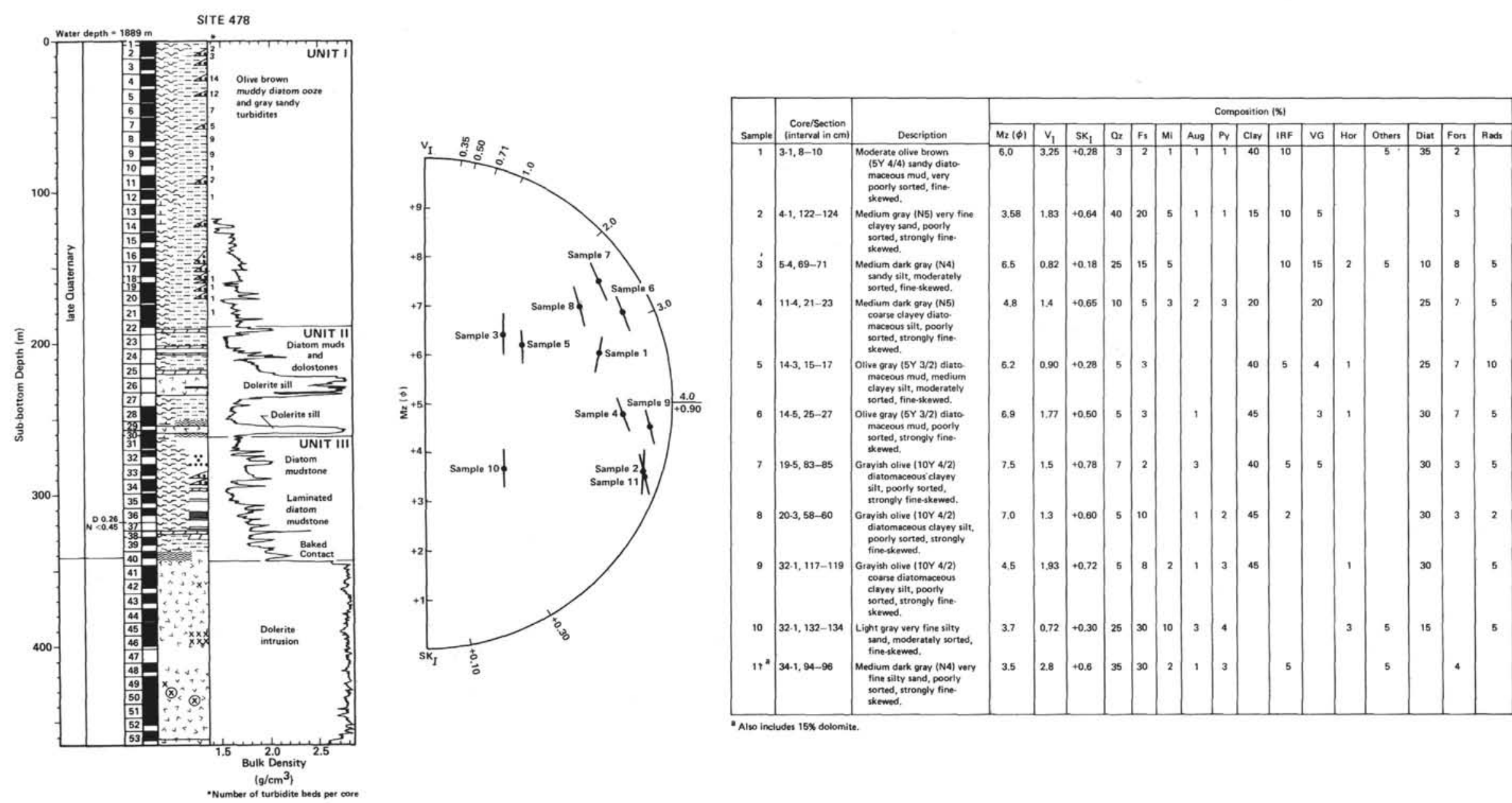

Figure 5. Mineralogical and textural attributes of terrigenous sediments from Hole 478. (See Fig. 2 for explanation of symbols and abbreviations.) 


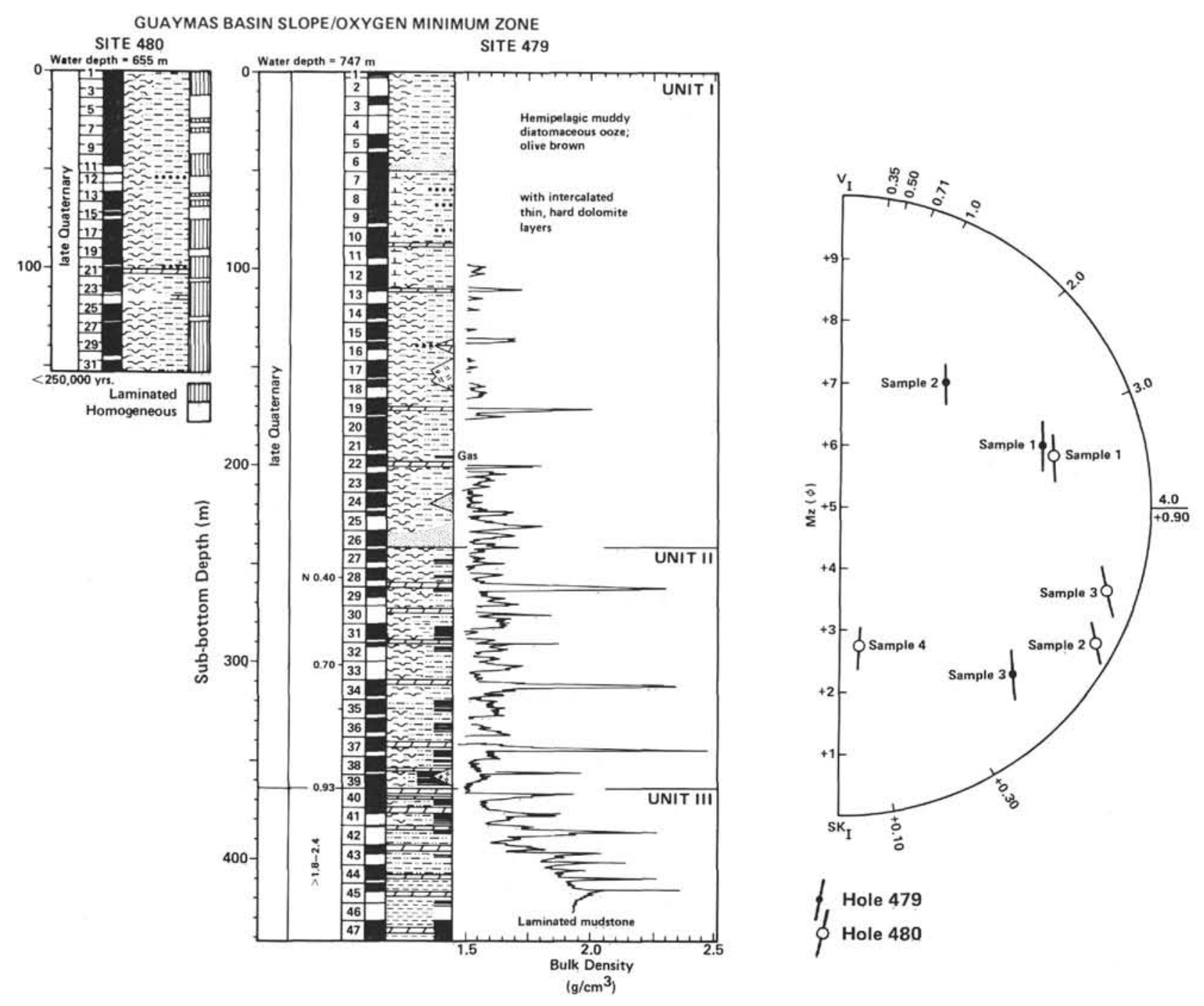

\begin{tabular}{|c|c|c|c|c|c|c|c|c|c|c|c|c|c|c|c|c|c|c|c|c|c|c|}
\hline \multirow[b]{2}{*}{ Sample } & \multirow{2}{*}{$\begin{array}{r}\text { Core/Section } \\
\text { (interval in cm) }\end{array}$} & \multirow[b]{2}{*}{ Description } & \multicolumn{20}{|c|}{ Composition (\%) } \\
\hline & & & $\operatorname{Mz}(\phi)$ & $v_{1}$ & $\mathrm{SK}_{\mathrm{I}}$ & $\mathrm{Oz}$ & $F_{s}$ & Mi & Aug & IRF & Py & vg & Hor & Epid & Apat & Hyp & Clay & Others & Diat & Fors & Rads & Sp Spic \\
\hline 1 & $\mid \begin{array}{l}479 \\
6 \cdot 6,32-35\end{array}$ & $\begin{array}{l}\text { Moderate olive brown (5Y 4/4) } \\
\text { and grayish (N5) muddy } \\
\text { diatomaceous ooze, poorly } \\
\text { sorted, strongly fine. } \\
\text { skewed. }\end{array}$ & 6.0 & 1.80 & +0.40 & 15 & 30 & 5 & 5 & 10 & 5 & & & & & & 10 & & 20 & & & \\
\hline 2 & 26.1, 137-139 & $\begin{array}{l}\text { Olive gray (5Y } 3 / 2 \text { ) and } \\
\text { grayish (N5) clavey silt, } \\
\text { moderately sorted, } \\
\text { fine-skewed. }\end{array}$ & 7.0 & 0.85 & +0.22 & 20 & 15 & & 2 & 5 & 1 & 2 & & & & & 40 & & 10 & 5 & & \\
\hline 3 & $26-3,107-109$ & $\begin{array}{l}\text { Dark greenish gray (5G 4/1) } \\
\text { muddy fine sand, poorly } \\
\text { sorted, strongly fine- } \\
\text { skewed. }\end{array}$ & 2.23 & 1.16 & +0.33 & 15 & 20 & & & 15 & 1 & 2 & & & & & 40 & & 3 & 2 & 1 & 1 \\
\hline $1^{a}$ & $\begin{array}{l}480 \\
12, \mathrm{cc}\end{array}$ & $\begin{array}{l}\text { Olive gray (5Y 3/2) clayey } \\
\text { silt, poorly sorted, } \\
\text { strongly fine-skewed. }\end{array}$ & 5.75 & 1.80 & +0.48 & 20 & 30 & 3 & 5 & 10 & 5 & 3 & 2 & & & & 15 & & 5 & & & \\
\hline 2 & $20-1,10$ & $\begin{array}{l}\text { Medium gray (N5) fine sand, } \\
\text { poorly sorted, strongly } \\
\text { fine-skewed. }\end{array}$ & 2.75 & 1.40 & +0.55 & 20 & 50 & 5 & 3 & 10 & & 5 & 1 & 1 & 3 & 2 & & & & & & \\
\hline 3 & $20-1,103-104$ & $\begin{array}{l}\text { Greenish gray (5G } 4 / 1 \text { ) very } \\
\text { fine sand, voorly sorted, } \\
\text { strongly fine-skewed. }\end{array}$ & 3.56 & 1.68 & +0.61 & 25 & 40 & 5 & 5 & 5 & 2 & 3 & 3 & & 2 & 3 & & 7 & & & & \\
\hline 4 & $31 \cdot 1,89-99$ & $\begin{array}{l}\text { Greenish gray (5G 4/1) fine } \\
\text { sand, well-sorted, near } \\
\text { symmetrical. }\end{array}$ & 2.69 & 0.49 & +0.01 & 25 & 45 & 10 & 3 & 5 & & 5 & 1 & & 1 & & & 5 & & & & \\
\hline
\end{tabular}

- Also includes $2 \%$ hematite.

Figure 6. Mineralogical and textural attributes of terrigenous sediments from Holes 479 and 480 . Note predominance of feldspar. (See Fig. 2 for explanation of symbols and abbreviations.) 


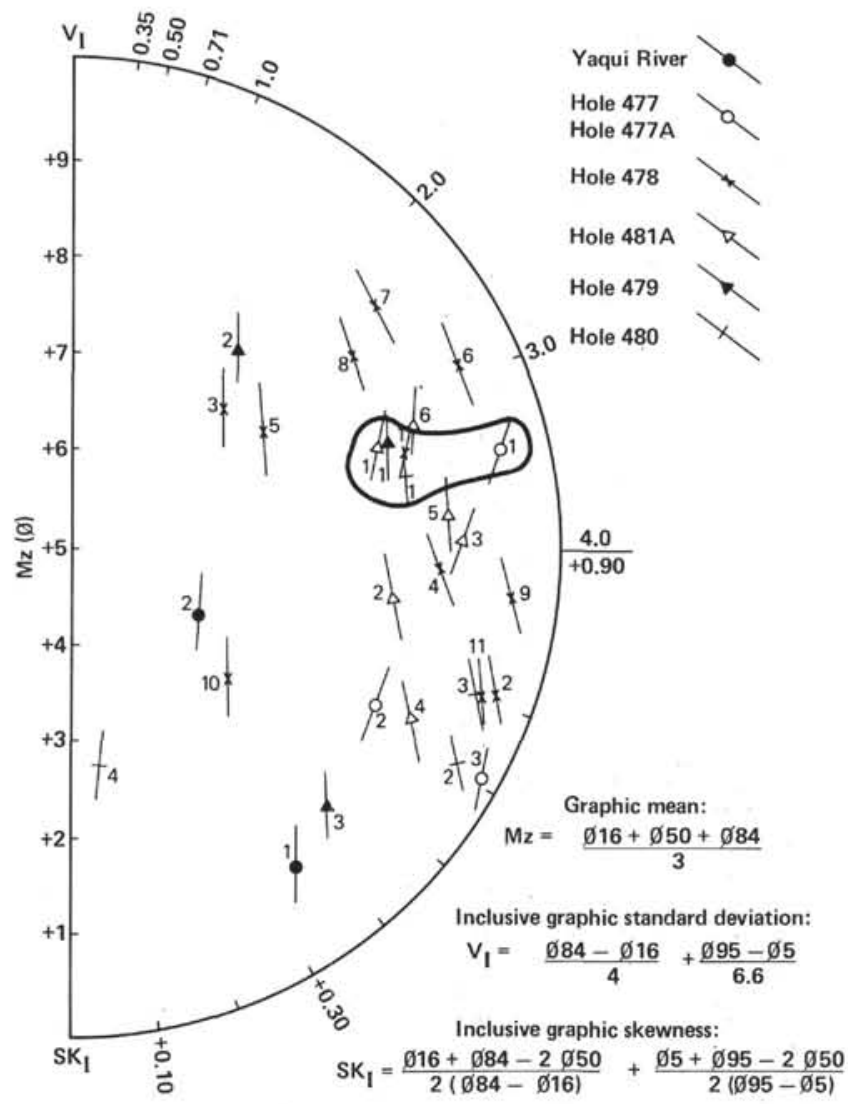

Figure 7. Textural attributes of terrigenous sediments for all samples from the Guaymas Basin and from the Yaqui River. (Numbers refer to sample locations on lithology for Figures 2-6.)

Table 1. Mineral composition (total percent) for Leg 64 sites in the Guaymas Basin.

\begin{tabular}{|c|c|c|c|c|c|c|c|c|c|c|c|c|c|c|c|c|c|c|c|c|c|c|c|c|c|}
\hline Sample & Location & $Q z$ & Fs & IRF & vG & Mi & Aug & Hor & Epid & Hyp & Apat & Oliv & Zeol & Mag & Tit & $\mathrm{Ilm}$ & Py & Hem & Dol & Diat & Rads & Fors & Sp Spic & Clay & Others \\
\hline 1 & Yaqui River & 25 & 15 & 20 & & 10 & 5 & 5 & 5 & 3 & 3 & 3 & & 3 & 3 & & & & & & & & & & \\
\hline 2 & Yaqui River & 45 & 30 & & & 10 & & 4 & 3 & & 5 & & & 3 & & 2 & & & & & & & & & \\
\hline 1 & $477-4-1,100-102 \mathrm{~cm}$ & 5 & 2 & & & 2 & 1 & & & & & & & & & & 5 & & & 45 & 15 & 10 & 15 & & \\
\hline 2 & $477 \mathrm{~A}-5-4,106-108 \mathrm{~cm}$ & 30 & 35 & & 10 & & & & 15 & & & & & & & & 2 & & & & & 8 & & & \\
\hline 3 & $477 \mathrm{~A}-9-1,53-55 \mathrm{~cm}$ & 40 & 20 & & & & & 10 & 10 & & & & 15 & & & & 2 & 3 & & & & & & & \\
\hline 1 & $478-3-1,8-10 \mathrm{~cm}$ & 3 & 2 & 10 & & 1 & 1 & & & & & & & & 1 & & & & & 35 & & 2 & & 40 & 6 \\
\hline 2 & $478-4-1,122-124 \mathrm{~cm}$ & 40 & 20 & 10 & 5 & 5 & $i$ & & & & & & & & & & I & & & & & 3 & & 15 & \\
\hline 3 & $478-5-4,69-71 \mathrm{~cm}$ & 25 & 15 & 10 & 15 & 5 & & 2 & & & & & & & & & & & & 10 & 5 & 8 & & & 5 \\
\hline 4 & $478-11-4,21-23 \mathrm{~cm}$ & 10 & 5 & & 20 & 3 & 2 & & & & & & & & & & 3 & & & 25 & 5 & 7 & & 20 & \\
\hline 5 & $478-14-3,15-17 \mathrm{~cm}$ & 5 & 3 & 5 & 4 & & & 1 & & & & & & & & & & & & 25 & 10 & 7 & & 40 & \\
\hline 6 & $478-14-5,25-27 \mathrm{~cm}$ & 5 & 3 & & 3 & & 1 & i & & & & & & & & & & & & 30 & 5 & 7 & & 45 & \\
\hline 7 & $478-19-5,83-85 \mathrm{~cm}$ & 7 & 2 & 5 & 5 & & 3 & & & & & & & & & & & & & 30 & 5 & 3 & & 40 & \\
\hline 8 & $478-20-3,58-60 \mathrm{~cm}$ & 5 & 10 & 2 & & & $i$ & & & & & & & & & & 2 & & & 30 & 2 & 3 & & 45 & \\
\hline 9 & $478-32-1,117-119 \mathrm{~cm}$ & 5 & 8 & & & 2 & $i$ & 1 & & & & & & & & & 3 & & & 30 & 5 & & & 45 & \\
\hline 10 & $478-32-1,132-134 \mathrm{~cm}$ & 25 & 30 & & & 10 & 3 & 3 & & & & & & & & & 4 & & & 15 & 5 & & & & 5 \\
\hline 11 & $478-34-1,94-96 \mathrm{~cm}$ & 35 & 30 & 5 & & 2 & 1 & & & & & & & & & & 3 & & 15 & 4 & & & & & 6 \\
\hline 1 & $481 \mathrm{~A}-1-1,60-62 \mathrm{~cm}$ & 3 & 5 & & & 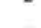 & $i$ & 1 & & & & & & & & & 2 & & & 30 & 10 & 3 & 5 & 40 & \\
\hline 2 & $481 \mathrm{~A}-1-2,96-99 \mathrm{~cm}$ & 10 & 20 & 5 & & & & & & & & & & & & & 3 & & & 20 & & $i$ & & 40 & 1 \\
\hline 3 & $481 \mathrm{~A}-2-1,113-115 \mathrm{~cm}$ & 10 & 15 & 2 & & 2 & & 1 & & & & & & & & & 2 & & & 16 & 1 & 1 & 3 & 45 & 3 \\
\hline 4 & $481 \mathrm{~A}-6-4,63-65 \mathrm{~cm}$ & 30 & 35 & 2 & & 3 & 2 & & & & & & & & & & $i$ & & & is & 2 & 5 & & & 5 \\
\hline 5 & $481 \mathrm{~A}-6-5,78-80 \mathrm{~cm}$ & 30 & 35 & $i$ & & 2 & & 2 & & & & & & & & & 3 & & & 20 & $i$ & 1 & & & 5 \\
\hline 6 & $481 \mathrm{~A}-12-3,19-21 \mathrm{~cm}$ & 20 & 25 & 7 & 3 & 3 & 5 & 5 & & & & & & & & & & & & 3 & 2 & 5 & & 20 & 2 \\
\hline 1 & $479-6-6,33-35 \mathrm{~cm}$ & 15 & 30 & 10 & & 5 & 5 & & & & & & & & & & 5 & & & 20 & & & & 10 & \\
\hline 2 & $479-26-1,137-139 \mathrm{~cm}$ & 20 & 15 & 5 & 2 & & 2 & & & & & & & & & & 1 & & & 10 & & 5 & & 40 & \\
\hline 3 & $479-26-3,107-109 \mathrm{~cm}$ & 15 & 20 & 15 & 2 & & & & & & & & & & & & $i$ & & & 3 & 1 & 2 & 1 & 40 & \\
\hline i & $480-12, \mathrm{CC}$ & 20 & 30 & 10 & 3 & 3 & 5 & 2 & & & & & & & & & 5 & 2 & & 5 & & & & 15 & \\
\hline 2 & $480-20-1,10 \mathrm{~cm}$ & 20 & 50 & 10 & 5 & 5 & 3 & $i$ & 1 & 2 & 3 & & & & & & & & & & & & & & \\
\hline 3 & $480-20-1,103-114 \mathrm{~cm}$ & 25 & 40 & 5 & 3 & 5 & 5 & 3 & & 3 & 2 & & & & & & 2 & & & & & & & & 7 \\
\hline 4 & $480-31-1,89-99 \mathrm{~cm}$ & 25 & 45 & 5 & 5 & 10 & 3 & 1 & & 1 & & & & & & & & & & & & & & & 5 \\
\hline
\end{tabular}

Note: See Figure 2 for explanations of abbreviations. 